American Journal of Applied Sciences 5 (1): 48-54, 2008

ISSN 1546-9239

(C) 2008 Science Publications

\title{
MINLP Optimization of Mechanical Structures
}

\author{
${ }^{1}$ S. Šilih, ${ }^{1}$ T. Žula, ${ }^{2}$ Z. Kravanja and ${ }^{1}$ S. Kravanja \\ ${ }^{1}$ University of Maribor, Faculty of Civil Engineering, \\ Smetanova 17, 2000 Maribor, Slovenia \\ ${ }^{2}$ University of Maribor, Faculty of Chemistry and Chemical Engineering, \\ Smetanova 17, 2000 Maribor, Slovenia
}

\begin{abstract}
We presented optimization of mechanical structures, performed by the Mixed-Integer Nonlinear Programming (MINLP) optimization approach. The MINLP is a combined continuous/discrete optimization technique, where a structural topology and standard/discrete sizes are optimized simultaneously with the continuous variables. Ddiscrete binary 0-1 variables were used to express the discrete decisions. For solution of this non-linear, continuous/discrete and non-convex MINLP class of the optimization problem, Modified Outer-Approximation/Equality-Relaxation (OA/ER) algorithm was used. A two-phase MINLP strategy applied for the optimization to accelerate the convergence of the mentioned algorithm.
\end{abstract}

Keywords: $\quad$ Mixed-Integer Non-linear programming, MINLP, Topology optimization, Shape optimization, Discrete sizes optimization.

\section{INTRODUCTION}

The paper discusses the optimization of mechanical structures, performed by the Mixed-Integer Non-linear Programming approach (MINLP). The MINLP is a combined continuous/discrete optimization technique. It handles with continuous and discrete binary 0-1 variables simultaneously. While continuous variables are defined for the continuous optimization of parameters (dimensions, stresses, strains, weights, costs.), discrete variables are used to express discrete decisions, i.e. usually the existence or non-existence of structural elements inside the defined structure. Different standard/discrete sizes may also be defined as discrete alternatives. Since the MINLP performs continuous and discrete optimizations simultaneously, the MINLP approach also finds optimal continuous parameters (mass, costs, stresses), a structural topology (with an optimal number and a configuration of structural elements) and discrete standard sizes simultaneously.

The MINLP optimization approach is proposed to be performed through three steps. The first one includes the generation of a mechanical superstructure of different topology and standard dimension alternatives, the second one involves the development of an MINLP model formulation and the last one consists of a solution for the defined MINLP optimization problem. The MINLP continuous/discrete optimization problems of structural optimization are in most cases comprehensive, non-convex and highly non-linear. The Outer-Approximation/Equality-Relaxation (OA/ER) algorithm ${ }^{[1-2]}$ is thus used. A two-phase MINLP optimization is proposed to accelerate the convergence of the mentioned algorithm. The optimizations are carried out by the MINLP computer package MIPSYN, the successor of PROSYN ${ }^{[1]}$ and TOP ${ }^{[2-4]}$.

Three examples are presented at the end of the paper. The first one introduces the topology, shape and sizing optimization of an aluminium truss cantilever, the second example shows topology and discrete/standard sizing optimization of a $40 \mathrm{~m}$ long steel truss and the last one presents the topology and standard sizing optimization of a single-storey industrial steel building.

\section{MATERIALS AND METHODS}

Mechanical superstructure: The MINLP optimization approach requires the generation of an MINLP mechanical superstructure composed of various topology and design alternatives that are all candidates

Corresponding Author: $\quad$ Stojan Kravanja, University of Maribor, Faculty of Civil Engineering, Smetanova 17, Postal Code: SI-2000, Maribor, Slovenia 
for a feasible and optimal solution. While topology alternatives represent different selections and interconnections of corresponding structural elements, design alternatives include different standard dimensions.

The superstructure is typically described by means of unit representation: i.e. structural elements and their interconnection nodes. Each potential topology alternative is represented by a special number and a configuration of selected structural elements and their interconnections; each structural element may in addition have different standard dimension alternatives. The main goal is thus to find within the given superstructure a feasible structure that is optimal with respect to topology, standard dimensions and all defined continuous parameters.

MINLP model formulation: It is assumed that a general non-linear and non-convex continuous/discrete optimization problem can be formulated as an MINLP problem in the form:

$$
\begin{gathered}
\min z=\boldsymbol{c}^{\mathrm{T}} \boldsymbol{y}+f(\boldsymbol{x}) \\
\text { s.t.: } \\
\boldsymbol{h}(\boldsymbol{x})=\mathbf{0} \\
\boldsymbol{g}(\boldsymbol{x}) \leq \mathbf{0} \\
\boldsymbol{B} \boldsymbol{y}+\boldsymbol{C} \boldsymbol{x} \leq \boldsymbol{b} \\
\boldsymbol{x} \in X=\left\{\boldsymbol{x} \in R^{\text {n }}: \boldsymbol{x}^{\text {lo }} \leq \boldsymbol{x} \leq \boldsymbol{x}^{\text {up }}\right\}
\end{gathered}
$$

(MINLP)

where $\boldsymbol{x}$ is a vector of continuous variables specified in the compact set $X$ and $y$ is a vector of discrete, binary 0 1 variables. Functions $f(\boldsymbol{x}), \boldsymbol{h}(\boldsymbol{x})$ and $\boldsymbol{g}(\boldsymbol{x})$ are non-linear functions involved in the objective function $z$, equality and inequality constraints, respectively. All functions $f(x), \quad \boldsymbol{h}(\boldsymbol{x})$ and $\boldsymbol{g}(\boldsymbol{x})$ must be continuous and differentiable. Finally, $\boldsymbol{B} \boldsymbol{y}+\boldsymbol{C} \boldsymbol{x} \leq \boldsymbol{b}$ represents a subset of mixed linear equality/inequality constraints.

The above general MINLP model formulation has been adapted for structural optimization. It is postulated that it helps us construct a MINLP mathematical optimization model for any structure.

In the context of structural optimization, continuous variables $\boldsymbol{x}$ define structural parameters (dimensions, strains, stresses, costs, mass...) and binary variables $y$ represent the potential existence of structural elements within the defined superstructure. An extra binary variable $y$ is assigned to each structural element. The element is then selected to compose the structure if its subjected binary variable takes value one $(y=1)$, otherwise it is rejected $(y=0)$. Binary variables also define the choice of discrete/standard sizes.

The economical (or mass) objective function $z$ involves fixed costs (mass) in the term $\boldsymbol{c}^{T} \boldsymbol{y}$, while the dimension dependant costs (mass) are included in the function $f(\boldsymbol{x})$. Non-linear equality and inequality constraints $\boldsymbol{h}(\boldsymbol{x})=\mathbf{0}$, $\boldsymbol{g}(\boldsymbol{x}) \leq \mathbf{0}$ and the bounds of the continuous variables represent the rigorous system of the design, loading, resistance, stress, deflection, constraints known from the structural analysis. Logical constraints that must be fulfilled for discrete decisions and structure configurations, which are selected from within the superstructure, are given by $\boldsymbol{B} \boldsymbol{y}+\boldsymbol{C} \boldsymbol{x} \leq \boldsymbol{b}$. These constraints describe relations between binary variables and define the structure's topology, materials and standard dimensions. It should be noted, that the comprehensive MINLP model formulation for mechanical structures may be found elsewhere ${ }^{[3,5]}$.

MINLP methods: A general MINLP class of optimization problem can be solved in principle by the following algorithms and their extensions:

-the Nonlinear Branch and Bound, NBB, proposed and used by many authors, e.g. E.M.L. Beale ${ }^{[6]}$, O.K. Gupta and A. Ravindra ${ }^{[7]}$;

-the Sequential Linear Discrete Programming method, SLDP, by G.R. Olsen and G.N. Vanderplaats ${ }^{[8]}$ and M. Bremicker et al. ${ }^{[9]}$;

-the Extended Cutting Plane method by T. Westerlund and F. Pettersson ${ }^{[10]}$;

-Generalized Benders Decomposition, GBD, by J.F. Benders $^{[11]}$, A.M. Geoffrion ${ }^{[12]}$;

-the Outer-Approximation/ Equality-Relaxation algorithm, OA/ER, by G.R. Kocis and I.E. Grossmann ${ }^{[13]}$;

-the Feasibility Technique by H. Mawengkang and B.A. Murtagh $^{[14]}$; and

-the LP/NLP based Branch and Bound algorithm by I. Quesada and I.E. Grossmann ${ }^{[15]}$.

Modified OA/ER algorithm: The OA/ER algorithm consists of solving an alternative sequence of Nonlinear Programming (NLP) optimization subproblems and Mixed-Integer Linear Programming (MILP) master problems, see Fig. 1. The former corresponds to continuous optimization of parameters for a mechanical structure with a fixed topology (and fixed discrete/standard dimensions) and yields an upper bound to the objective to be minimized. The latter involves a global approximation to the superstructure of alternatives in which a new topology and discrete/standard dimensions are identified so that its lower bound does not exceed the current best upper 
bound. The search of a convex problem is terminated when the predicted lower bound exceeds the upper bound, otherwise it is terminated when the NLP solution can not be improved. The OA/ER algorithm guarantees the global optimality of solutions for convex and quasi-convex optimization problems.

The OA/ER algorithm as well as all other mentioned MINLP algorithms do not generally guarantee that the solution found is the global optimum. This is due to the presence of nonconvex functions in the models that may cut off the global optimum. In order to reduce undesirable effects of nonconvexities the Modified OA/ER algorithm was proposed by $Z$. Kravanja and I.E. Grossmann ${ }^{[1]}$, see also S. Kravanja et al. ${ }^{[2]}$, by which the following modifications are applied for the master problem: the deactivation of linearizations, the decomposition and the deactivation of the objective function linearization, the use of the penalty function, the use of the upper bound on the objective function to be minimized as well as the global convexity test and the validation of the outer approximations.

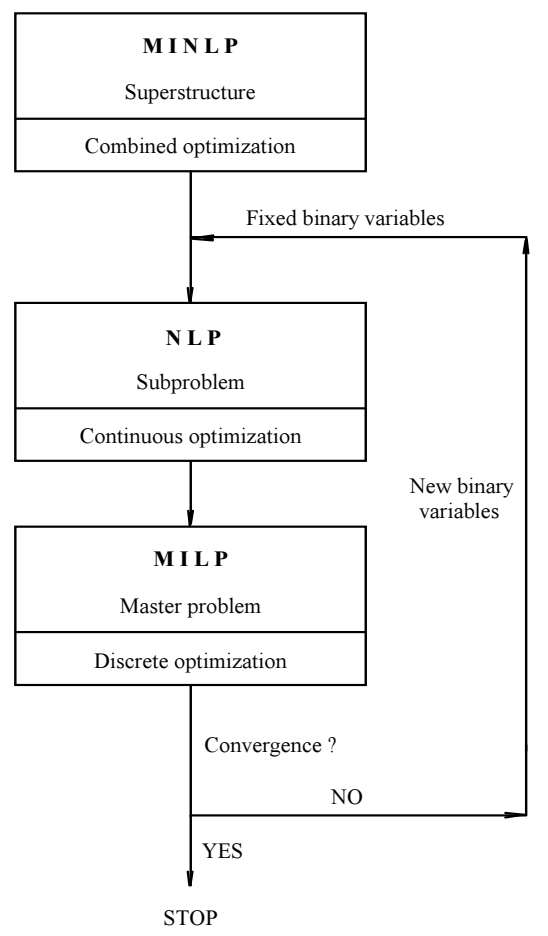

Fig. 1: Steps of the OA/ER algorithm

Two-phase MINLP optimization: The optimal solution of complex non-convex and non-linear MINLP problem with a high number of discrete decisions is in general very difficult to be obtained. The optimization is thus proposed to be performed sequentially in two different phases to accelerate the convergence of the OA/ER algorithm. The optimization starts with the topology optimization of a structure, while discrete sizes are relaxed temporary into continuous parameters. When the optimal topology is found, discrete sizes are in the second phase re-established and the simultaneous topology and discrete sizing optimization of the structure is then continued until the optimal solution is found.

\section{RESULTS AND DISCUSSION}

MINLP optimization approach is illustrated by three examples. The first one introduces the topology, shape and sizing optimization of an aluminium truss cantilever, the second example shows the topology and discrete/standard sizing optimization of a $40 \mathrm{~m}$ long steel truss and the last one presents the topology and discrete/standard sizing optimization of a single-storey industrial steel building.

The optimization of the structures is proposed to be carried out by a user-friendly version of the MINLP computer package MIPSYN, the successor of PROSYN $^{[1]}$ and TOP ${ }^{[2-4,}{ }^{16]}$. MIPSYN is the implementation of many advanced optimization techniques, most important of which are the Modified OA/ER algorithm and MINLP strategies. In terms of complexity, the MIPSYN's synthesis problems can range from a simple NLP optimization problem of a single structure up to the MINLP optimization of a complex superstructure problem. MIPSYN runs automatically or in an interactive mode and thus provides the user with a good control and supervision of the calculations. GAMS/CONOPT2 ${ }^{[17]}$ (Generalized reduced-gradient method) is used to solve NLP subproblems and GAMS/Cplex 7.0 $0^{[18]}$ (Branch and Bound) is used to solve MILP master problems.

For each type of structure, a special optimization model must be developed. Each model is constructed on the basis of the mentioned general MINLP-G model formulation. As an interface for mathematical modelling and data inputs/outputs GAMS (General Algebraic Modelling System) ${ }^{[19]}$, a high level language, is used.

Topology, shape and sizing optimization of an aluminium truss cantilever: The first numerical example presents the topology, shape and sizing optimization of an aluminium truss cantilever. The superstucture of the cantilever comprises 14 joints and 
30 alternative elements. It is shown in Fig. 2. The input data are: $L=500 \mathrm{~cm}, H=1050 \mathrm{~cm}, q_{\mathrm{sd}}=10 \mathrm{kN} / \mathrm{m}$, Young's modulus $E=68.97 \mathrm{GPa}$, the limit tensile/compressive stress $\sigma^{\downarrow, \max }=\sigma^{\mathcal{c}, \max }=172.4 \mathrm{MPa}$ and the allowed vertical displacements of unsupported joints are $v_{i}^{\max }=15 \mathrm{~cm}$. The design uniformly distributed load $q_{\mathrm{Sd}}$ is approximated to the nodal forces acting on the joints of the bottom chord.

The MINLP optimization model ALUMTRUSS was developed for the optimization. The objective function defines the weigth of the structure. The unit weigth of the aluminium material amouts to 27126.4 $\mathrm{N} / \mathrm{m}^{3}$. The objective function is subjected to the set of constraints known from structural analysis. The finite element equations were defined for the calculation of internal forces and displacements. Design constraints are defined in order to check the tensile and compressive/buckling resistance of bars as well as vertical displacements of joints. The limit buckling stress of a compressed element is considered as equal to the Euler buckling stress for circular cross-sections.

For each element, a binary variable $y_{i, j}$ is introduced in order to define the existance or nonexistance of a bar inside the current structural topology, where $i$ and $j$ stand for the initial and the end joint of the element, respectively. A set of logical relations is then defined by which the kinematical stability of the truss is provided. The vertical $(\mathrm{Y})$ coordinates $y_{i}^{\mathrm{c}}$ of the top chord joints (joints 4, 6, 8, 10,12 and 14, see Fig. 2) are defined as continuous shape variables with their lower/upper bounds $y_{i}^{\mathrm{c}, \mathrm{LO}} / y_{i}^{\mathrm{c}, \mathrm{UP}}=100 / 1200 \mathrm{~cm}$. The cross section areas of bars $A_{i, j}$ are considered as continuous sizing variables. The lower/upper bounds on cross-sectional areas are $A_{i, j}{ }^{\mathrm{LO}} / A_{i, j}{ }_{\mathrm{UP}}=6.45 / 225.81 \mathrm{~cm}^{2}$.

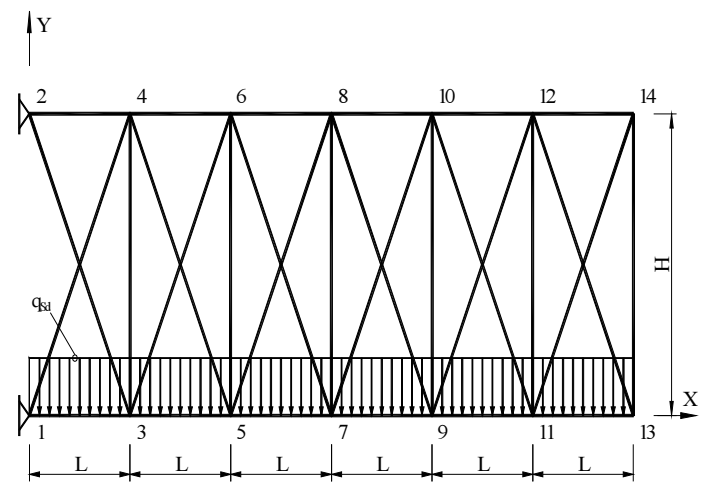

Fig. 2: Superstructure of the aluminium truss cantilever

The mixed continuous/discrete MINLP optimization was performed by the Modified OA/ER algorithm. The optimization model contained 248 mainly nonlinear (in)equality constraints, 146 continuous and 30 discrete/binary variables. The optimal solution was obtained at the $7^{\text {th }}$ MINLP iteration. The optimized structure is shown in Fig. 3. The obtained optimal continuous variables are listed in Table 1 .

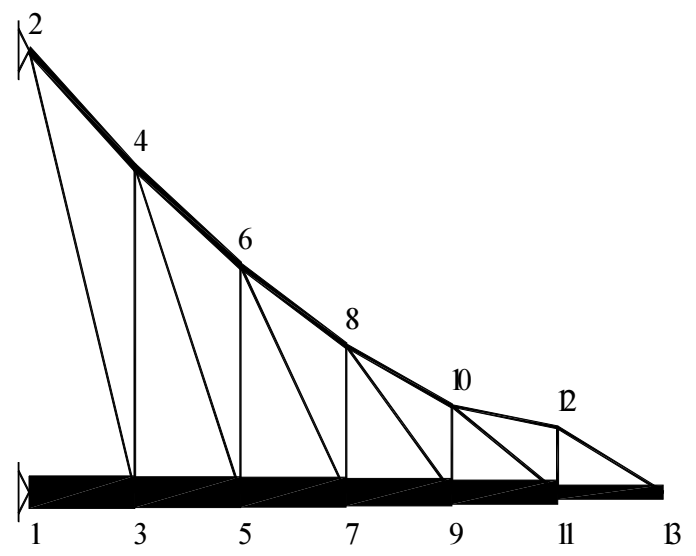

Fig. 3: The optimized aluminium truss cantilever structure

Topology and discrete/standard sizing optimization of a $40 \mathrm{~m}$ long steel truss: The second example presents the simultaneous topology and discrete/standard sizing optimization of a simply supported truss girder over the span of $40 \mathrm{~m}$, see Fig. 4 . The proposed superstructure includes 18 nodes and 41 alternative elements. The truss is subjected to a design uniformly distributed load $q_{\mathrm{Sd}}$ of $30 \mathrm{kN} / \mathrm{m}$, acting on the bottom chord. The uniform load is approximated to the nodal forces acting on the joints of the bottom chord. Truss elements are designed from standard circular hollow sections made of S 235 steel. The input data also include Young's modulus $E=210 \mathrm{GPa}$ and the unit mass of steel material $7850 \mathrm{~kg} / \mathrm{m}^{3}$.

The MINLP optimization model STEELTRUSS was developed for the optimization. The objective function of the structure's mass was subjected to the set of constraints known from structural analysis. The finite element equations were defined for the calculation of internal forces and displacements. Constraints for the dimensionig were defined in accordance with Eurocode $3^{[20]}$. Buckling lengths of the truss elements are considered as being equal to the system lengths of the elements for both in-plane and out-of-plane buckling.

The vectors of discrete/standard alternative values for the diameter $d$ and wall thickness $t$ of cross-sections are given as follows:

$\boldsymbol{d}=\{42.4,48.3,60.3,76.1,88.9,108.0,114.3$, 133.0, 139.7, 159.0, 168.3, 177.8, 193.7, 219.1, 244.5 $273.0\}[\mathrm{mm}]$ and

$\boldsymbol{t}=\{2.0,2.9,3.2,4.0,5.0,6.3,7.1,8.0,10.0,12.5$, $14.2,16.0\}[\mathrm{mm}]$. 
Table 1: The obtained optimal values of optimization variables

\begin{tabular}{|c|c|c|c|c|c|c|c|c|}
\hline \multicolumn{9}{|c|}{ Sizing variables } \\
\hline$A_{1,3}$ & $=$ & $76.55 \mathrm{~cm}^{2}$ & $A_{2,3}$ & $=$ & $6.45 \mathrm{~cm}^{2}$ & $A_{2,4}$ & $=$ & $20.36 \mathrm{~cm}^{2}$ \\
\hline$A_{3,4}$ & $=$ & $6.45 \mathrm{~cm}^{2}$ & $A_{3,5}$ & $=$ & $74.38 \mathrm{~cm}^{2}$ & $A_{4,5}$ & $=$ & $6.45 \mathrm{~cm}^{2}$ \\
\hline$A_{4,6}$ & $=$ & $17.57 \mathrm{~cm}^{2}$ & $A_{5,6}$ & $=$ & $6.45 \mathrm{~cm}^{2}$ & $A_{5,7}$ & $=$ & $71.30 \mathrm{~cm}^{2}$ \\
\hline$A_{6,7}$ & $=$ & $6.45 \mathrm{~cm}^{2}$ & $A_{6,8}$ & $=$ & $14.41 \mathrm{~cm}^{2}$ & $A_{7,8}$ & $=$ & $6.45 \mathrm{~cm}^{2}$ \\
\hline$A_{7,9}$ & $=$ & $66.51 \mathrm{~cm}^{2}$ & $A_{8,9}$ & $=$ & $6.45 \mathrm{~cm}^{2}$ & $A_{8,10}$ & $=$ & $10.26 \mathrm{~cm}^{2}$ \\
\hline$A_{9,10}$ & $=$ & $6.45 \mathrm{~cm}^{2}$ & $A_{9,11}$ & $=$ & $57.75 \mathrm{~cm}^{2}$ & $A_{10,11}$ & $=$ & $7.38 \mathrm{~cm}^{2}$ \\
\hline$A_{10,12}$ & $=$ & $6.45 \mathrm{~cm}^{2}$ & $A_{11,12}$ & $=$ & $8.52 \mathrm{~cm}^{2}$ & $A_{11,13}$ & $=$ & $33.36 \mathrm{~cm}^{2}$ \\
\hline \multirow{2}{*}{\multicolumn{9}{|c|}{$\begin{array}{c}A_{12,13}= \\
\text { Shape variables }\end{array}$}} \\
\hline & & & & & & & & \\
\hline$y_{4}{ }^{\mathrm{c}}$ & $=772.40 \mathrm{~cm}$ & & $y_{6}{ }^{\circ}$ & $=$ & $538.05 \mathrm{~cm}$ & $y_{8}{ }^{\mathrm{c}}$ & $=$ & $347.74 \mathrm{~cm}$ \\
\hline$y_{10}$ & $=204.99 \mathrm{~cm}$ & & $y_{1}$ & $=$ & $153.64 \mathrm{~cm}$ & & & \\
\hline \multicolumn{9}{|c|}{ Optimal weigth $W=5217.39 \mathrm{~N}$} \\
\hline
\end{tabular}

With respect to the available standard cross sections the lower and upper bound on wall thickness for each section diameter are defined. This way, 76 alternative standard cross-sections are defined, which can be attributed to each element of the truss. The cross sections of the chords are forced into being constant through the entire span. Since the loading of the defined truss is symmetric, symmetry of topology with respect to the vertical axis through the midspan of the structure is required.

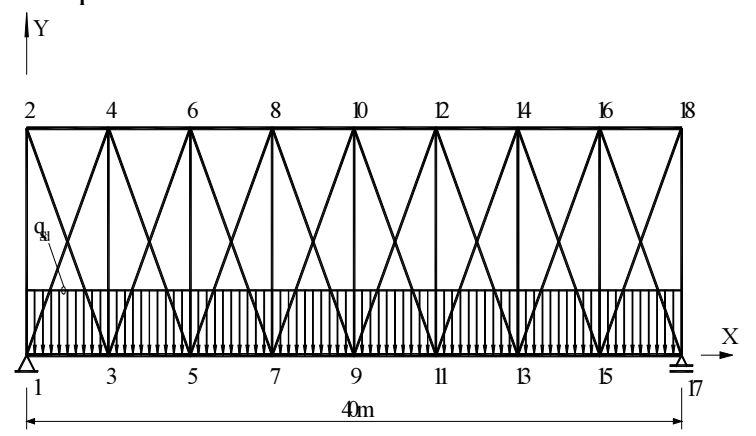

Fig. 4: Superstructure of the steel truss

The MINLP optimization of the structure's mass was performed by the computer package MIPSYN (GAMS/CONOPT2 and GAMS/Cplex 7.0). The Modified OA/ER algorithm and the two-phase MINLP optimization were applied. The optimization model contained 903 mainly nonlinear (in)equality constraints, 1629 continuous and 1189 discrete/binary variables. Fig. 5 shows the calculated optimal truss. The optimal mass yields $2544.995 \mathrm{~kg}$, obtained at the $51^{\mathrm{st}}$ main MINLP iteration. The obtained optimal height of the truss girder amouts to $767.82 \mathrm{~cm}$. The calculated optimal standard cross-section dimensions are listed in Table 2.

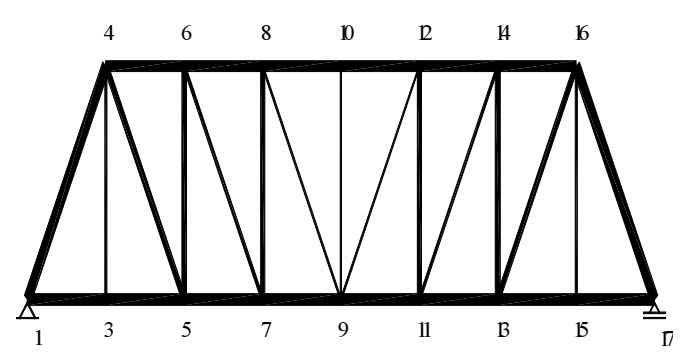

Fig. 5: Optimal steel truss

Topology and discrete/standard sizing optimization of an industrial steel building: The third example introduces the topology and discrete/standard sizing optimization of a single-storey industrial steel building. The building is 27 meters wide, 80 meters long and 6 meters high. The structure is consisted from equal nonsway steel portal frames, which are mutually connected with purlins, see Fig. 6 . Variable imposed loads $s=1.80$ $\mathrm{kN} / \mathrm{m}^{2}$ (snow), $w_{v}=0.2 \mathrm{kN} / \mathrm{m}^{2}$ (vertical wind) and $w_{h}=0.80 \mathrm{kN} / \mathrm{m}^{2}$ (total horizontal wind) are defined as the uniformly distributed surface load. The material used was steel S 355 . The task of the optimization was to find the minimal structure's mass, the optimal topology (the optimal number of portal frames and purlins) and all standard cross-sections.

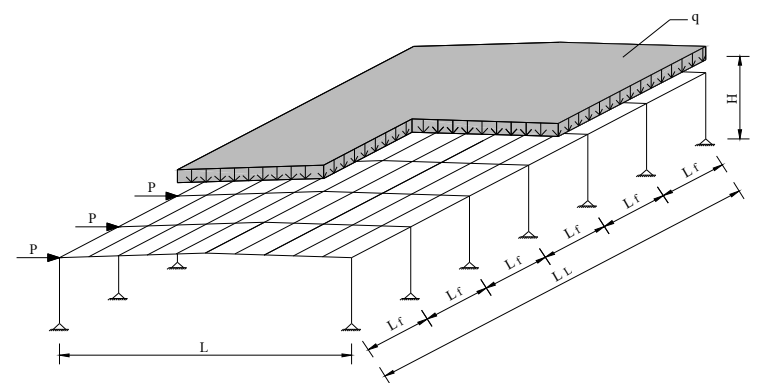

Fig. 6: Single-storey industrial steel building 
The MINLP optimization model FRAMEOPT was developed. The objective function of the structure's mass was defined. Both, the horizontal concentrated load $P$ at the top of the columns (wind) and the vertical uniformly distributed load $q$ on the frame beams (snow and wind) were calculated automatically through the optimization considering the calculated intermediate distance between the portal frames. Internal forces were calculated by the elastic first-order theory for the nonsway frame mode. The design of steel members was performed in accordance with Eurocode 3 for the conditions of both the ultimate and serviceability limit states.

Table 2: Optimal standard cross-sections

\begin{tabular}{ccc}
\hline Elemet & $\begin{array}{c}\text { Diameter } \\
d[\mathrm{~mm}]\end{array}$ & $\begin{array}{c}\text { Wall } \\
\text { thickness } t \\
{[\mathrm{~mm}]}\end{array}$ \\
\hline Bottom chord (1-17) & 193.7 & 4.0 \\
Top chord (4-16) & 177.8 & 5.0 \\
$1-4,16-17$ & 244.5 & 4.0 \\
$1-5,13-16,7-8,11-12$ & 133.0 & 2.0 \\
$3-4,15-16$ & 60.3 & 2.9 \\
$5-6,13-14$ & 159.0 & 3.2 \\
$6-7,11-14$ & 76.1 & 3.2 \\
$8-9,9-12,9-10$ & 42.4 & 2.0 \\
\hline
\end{tabular}

The industrial building superstructure was generated in which all possible structures were embedded by the topology variation of 30 portal frames and 20 purlins. The superstructure also comprised 24 different standard hot rolled European wide flange I sections, i.e. HEA sections (from HEA 100 to HEA 1000) for each column, beam and purlin separately.

The MINLP model of the industrial building contains 122 (in)equality constraints, 175 continuos and 112 discrete binary $0-1$ variables. The MINLP optimization of the structure's mass was performed by the computer package MIPSYN (GAMS/CONOPT2 and GAMS/Cplex 7.0). The Modified OA/ER algorithm and the two-phase MINLP optimization were applied. The optimal result of 172.68 tons was obtained in the $29^{\text {th }}$ main MINLP iteration. The optimal solution includes the obtained optimal topology of 18 portal frames and 14 purlins, see Fig. 7, as well as the optimal standard steel HEA sections of columns, beams and purlins, see Fig. 8.

\section{CONCLUSION}

The paper presents the Mixed-Integer Non-linear Programming (MINLP) approach to optimization of mechanical structures. The Modified OA/ER algorithm and the two-phase MINLP optimization strategy are

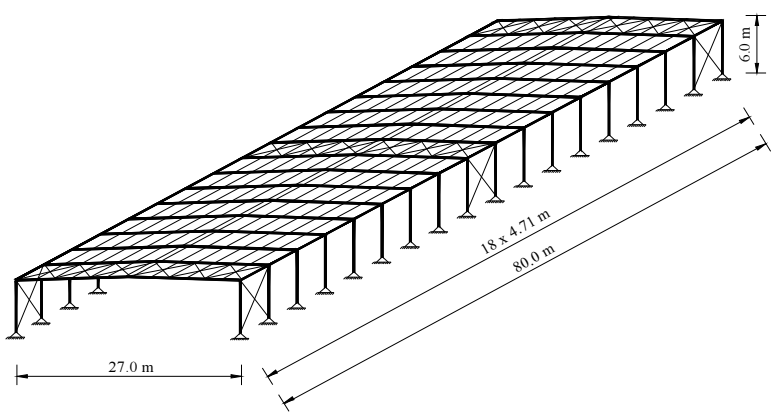

Fig. 7: Optimal structural topology of the singlestorey industrial building
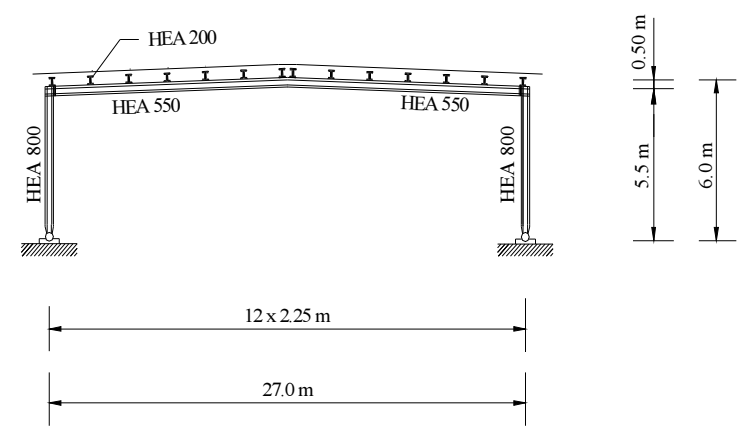

Fig. 8: Optimal steel sections

proposed to be applied. The two-phase optimization starts with the topology optimization of the structure, while standard dimensions are temporarily relaxed into continuous parameters. When the optimal topology is found, the standard dimensions of cross-sections are reestablished and the simultaneous topology and discrete/standard sizing optimization is then continued until the optimal solution is found. Beside the optimal structure's mass, the optimal topology with the optimal number of structure elements (bars, columns, beams, purlins), the optimal shape and the optimal discrete/standard cross-sectional sizes can be obtained simultaneously. Without performing the two-phase MINLP strategy no feasible or bad optimal results were obtained. Three examples, presented at the end of the paper, clearly show the efficiency of the proposed MINLP approach. 


\section{ACKNOWLEDGEMENTS}

This research was supported by the Slovenian Research Agency through research programme P2-0129 "Development, modelling and optimization of structures and processes in civil engineering and traffic".

\section{REFERENCES}

1. Kravanja, Z. and I.E. Grossmann, 1994. New Developments and Capabilities in PROSYN - An Automated Topology and Parameter Process Synthesizer. Computers \& Chemical Engineering, 18(11/12): 1097-1114.

2. Kravanja, S., Z. Kravanja and B. S. Bedenik, 1998. The MINLP optimization approach to structural synthesis, Part I: A general view on simultaneous topology and parameter optimization. International Journal for Numerical Methods in Engineering, 43(2): 263-292.

3. Kravanja, S., Z. Kravanja and B. S. Bedenik, 1998. The MINLP optimization approach to structural synthesis, Part II: Simultaneous topology, parameter and standard dimension optimization by the use of the Linked two-phase MINLP strategy. International Journal for Numerical Methods in Engineering, 43(2): 293-328.

4. Kravanja, S., Z. Kravanja and B. S. Bedenik, 1998. The MINLP optimization approach to structural synthesis, Part III: Synthesis of roller and sliding hydraulic steel gate structures. International Journal for Numerical Methods in Engineering, 43(2): 329-364.

5. Kravanja, S., S. Šilih and Z. Kravanja, 2005. The multilevel MINLP optimization approach to structural synthesis: the simultaneous topology, material, standard and rounded dimension optimization. Advances in Engineering Software, 36 (9): 568-583.

6. Beale, E.M.L., 1977. Integer Programming. In: The State of the Art in Numerical Analysis (ed D. Jacobs) pp. 409-448. Academic Press, London.

7. Gupta, O.K. and A. Ravindran, 1985. Branch and bound experiments in convex nonlinear integer programming. Management Science, 31(12): 1533-1546.

8. Olsen, G.R. and G.N. Vanderplaats, 1989. Method for Nonlinear Optimization with Discrete Design Variables. AIAA Journal, 27(11): 1584-1589.
9. Bremicker, M., P. Y. Papalambros and H.T. Loh, 1990. Solution of Mixed-Discrete Structural Optimization Problems with a New Sequental Linearization Method. Computers and Structures, 37(4): 451-461.

10. Westerlund, T. and F. Pettersson, 1995. An extended cutting plane method for solving convex MINLP problems, European Symposium on Computer Aided Process Engineering-5, Supplement to Computers \& Chemical Engineering, pp. 131-136. Bled, Slovenia.

11. Benders, J.F., 1962. Partitioning Procedures for Solving Mixed-variables Programming Problems. Numerische Mathematik, 4: 238-252.

12. Geoffrion, A.M., 1972. Generalized Benders Decomposition. Journal of Optimization Theory and Applications, 10(4): 237-260.

13. Kocis, G.R. and I.E. Grossmann, 1987. Relaxation Strategy for the Structural Optimization of Process Flowsheets. Industrial \& Engineering Chemistry Research, 26(9): 1869-1880.

14. Mawengkang, H. and B.A. Murtagh, 1986. Solving Nonlinear Integer Programs with Large-Scale Optimization Software. Annals of Operations Research, 5: 425-437.

15. Quesada, I. and I.E. Grossmann, 1992. An LP/NLP Based Branch and Bound Algorithm for Convex MINLP Optimization Problems. Computers \& Chemical Engineering, 16, 1992, pp. 937-947.

16. Kravanja, S., Z. Kravanja, B. S. Bedenik and S. Faith, 1992. Simultaneous Topology and Parameter Optimization of Mechanical Structures. In: Proceedings of the First European Conference on Numerical Methods in Engineering (eds C. Hirsch et al.) pp. 487-495. Elsevier, Amsterdam.

17. Drudd, A.S., 1994. CONOPT - A Large-Scale GRG Code. ORSA Journal on Computing, 6(2): 207-216.

18. GAMS/CPLEX 7.0 User Notes, 2001. ILOG inc.

19. Brooke, A., D. Kendrick, A. Meeraus and R. Ramesh, 1998. GAMS - A User's Guide. GAMS Development Corporation, Washington.

20. Eurocode 3: Design of steel structures, Part 1-1: General rules and rules for buildings, 1992. European Comitee for Standardization (CEN), Brussels. 